\title{
Blind Detection of Space-Time Coded Block CDMA Signals Based on the Minimum Variance Criterion: An Unified View and Performance Comparison
}

\author{
César A. Medina
}

\author{
Raimundo Sampaio-Neto
}

\begin{abstract}
Resumo-Neste trabalho propõe-se uma estrutura para transmissão e deteção de sinais em sistemas CDMA por blocos com codificação espaço-temporal. Considera-se os casos de transmissão em multiportadora e portadora única, ambos com intervalo de guarda do tipo preenchimento de zeros e do tipo prefixo cíclico. É também fornecida uma base comum para a análise unificada destes sistemas, de forma a permitir uma comparação eqüitativa entre eles. Nosso estudo enfoca o enlace direto e detetores às cegas baseados no critério de mínima variância, embora este mesmo modelo possa ser adaptado a um amplo conjunto de receptores. Resultados numéricos em relação a taxa de erro de bit (BER), a resistência ao efeito "perto-longe" (near-far resistance, NFR) e a robustez à carga do sistema são apresentados utilizando diferentes tipos de códigos de espalhamento.
\end{abstract}

Palavras-Chave-Transmissão CDMA por blocos, Mínima variância, MIMO, codificação espaço-temporal.

Abstract-In this paper, we propose a space-time block CDMA transmission system for downlink multipath channels. Multicarrier and single carrier transmission are considered, both with zero padding and cyclic prefix as guard intervals. We also provide a common ground for the analysis of these systems in order to allow an equal comparison between them. Our study focuses on blind detectors based on the minimum variance criterion, but this framework can be adapted to a wider range of receivers. Numerical results in terms of bit error rate (BER), near-far resistance (NFR) and robustness to system load are shown for different types of spreading codes.

Keywords-CDMA Block Transmission, Minimum Variance, MIMO, Space-time coding.

\section{INTRODUCTION}

A strong candidate for downlink multiple access in IEEE802.20 standard is multicarrier code division multiple access with cyclic prefix (MC CDMA CP) [1], which is based on the concatenation of direct sequence (DS) spreading and orthogonal frequency-division multiplexing (OFDM) transmission. The data symbol of a user is spread and the chips are simultaneously transmitted, each one over a narrowband subchannel by the multicarrier modulation (frequency domain spreading). The combination of direct sequence code division multiple access (DS-CDMA) and multicarrier (MC) modulation was first proposed in 1993 [2], [3].

César A. Medina and Raimundo Sampaio-Neto are with the Centro de Estudos em Telecomunicações (CETUC), Pontifícia Universidade do Rio de Janeiro (PUC-Rio), Rio de Janeiro (RJ), Brazil, E-mails: \{csmedina, raimundo\}@cetuc.puc-rio.br. This work was supported by the Brazilian Council for Scientific and Technological Development (CNPq).
Behind the importance of MC CDMA CP systems, in recent years, single carrier (SC) block transmission systems have also been widely studied in multiple user access schemes, such as code division multiple access (CDMA). Single carrier CDMA (SC CDMA) is the well known CDMA with a guard interval between each symbol [4], [5]. The chips are transmitted sequentially over the whole bandwidth allocated for that user. Both, single and multicarrier systems, have been proposed with cyclic prefix $(\mathrm{CP})$ and zero padding $(\mathrm{ZP})$ as guard intervals.

Blind adaptive linear receivers are promising techniques for interference suppression in CDMA-based systems, as they offer an attractive trade-off between performance and complexity and can be used in situations where the receiver loses track of the desired signal and a training sequence is not available. A blind adaptive detector for space-time single carrier DSCDMA systems in flat fading channels was introduced in [6]. It uses a Capon-like structure and requires only the knowledge of the spreading code and timing of the user to perform the detection. In [7] a constrained constant modulus receiver for space-time block coded single carrier DS-CDMA systems in frequency selective channels case is proposed and in [8] a MMSE receiver is proposed for single or multicarrier CDMA transmission in frequency selective channels. A blind receiver for MC CDMA CP was also proposed in [9].

In fourth generation standards, the use of the so-called multiple-input multiple-output (MIMO) systems is a common strategy. They make use of the multiple transmission channels formed between the transmitter and receiver, to send or receive signals and have gained a lot of attention as effective diversity techniques to combat fading and/or increase the capacity of wireless networks [10], [11] without increasing the bandwidth or power of the transmitted signal. This is achieved by a high spectral efficiency and robustness to fading. The increase in the wireless network capacity is achieved in the uplink through the use of space diversity multiple access (SDMA). In the mobile unit, the robustness to fading is achieved with the use of diversity techniques such as space-time coding [10], [11]. One of such techniques is the Alamouti space-time coding [12] which uses two transmit and multiple receive antennas.

In this work we propose a structure for space-time coded block CDMA transmission systems in frequency selective channels. We consider four commonly refereed systems, namely, multicarrier CDMA with cyclic prefix (MC CDMA $\mathrm{CP}$ ), multicarrier CDMA with zero padding (MC CDMA ZP), 
single carrier CDMA with cyclic prefix (SC CDMA CP) and single carrier CDMA with zero padding (SC CDMA ZP). We also provide a common ground in order to allow a fair comparison between the aforementioned systems. We restrict our study to blind detectors based on the minimum variance criterion, but this framework can be adapted to a wider range of receivers. Bit error rate (BER), near-far resistance (NFR) effect and robustness to system load results are shown.

This paper is structured as follows. Section II describes the proposed space-time block CDMA system model and introduces a generalized system framework. In Section III the proposed constrained minimum variance receiver is introduced and in Section IV a recursive least squares implementation is presented. Some simulation results are presented in Section V, while Section VI gives the conclusions.

\section{System MODEL}

A discrete model of the proposed block CDMA space-time coded system employing Alamouti's [12] scheme operating in frequency selective channels is depicted in Fig. 1. The
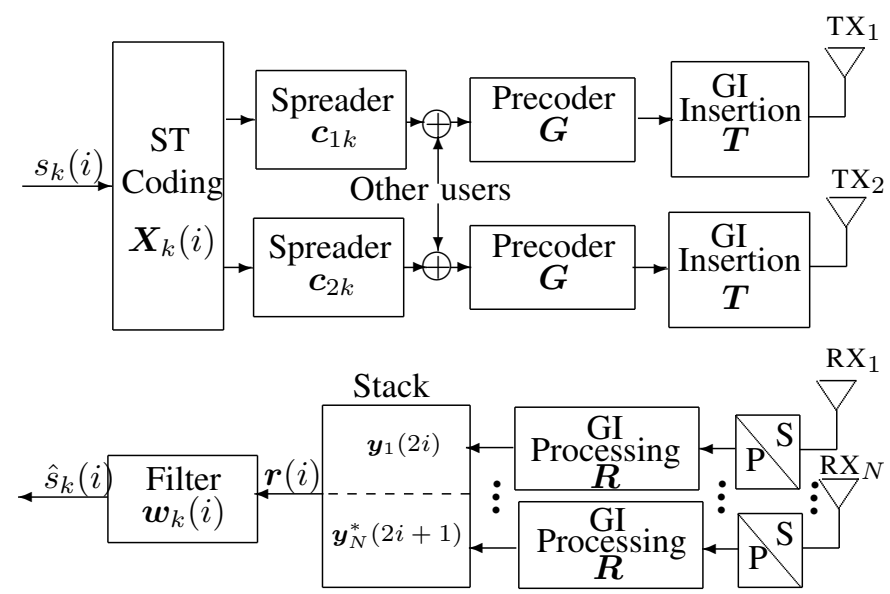

Fig. 1. Alamouti block CDMA transmission system.

proposed scheme uses two transmit and $N$ receive antennas. The symbols are first space-time coded by the space-time encoder, $\boldsymbol{X}_{k}(i)$, which maps the symbols of user $k$ according to

$$
\boldsymbol{X}_{k}(i)=\sqrt{\rho_{k}}\left[\begin{array}{cc}
s_{k}(2 i) & -s_{k}^{*}(2 i+1) \\
s_{k}(2 i+1) & s_{k}^{*}(2 i)
\end{array}\right]
$$

where $\rho_{k}=E_{k} / 2, E_{k}$ is the transmitted energy for user $k$, and $(\cdot)^{*}$ denotes complex conjugate. Different rows of $\boldsymbol{X}_{k}(i)$ refer to different branches of the transmitter (see Fig. 1) whereas different columns refer to different symbol periods. It is assumed that symbols $s_{k}(i)$, drawn from some constellation with zero mean and unit average symbol energy, are independent and identically distributed (i.i.d.).

Two spreading codes $\boldsymbol{c}_{j k}, \boldsymbol{c}_{j k}^{H} \boldsymbol{c}_{j k}=1, j=1,2$, of $M$ chips per symbol are assigned to each user, one for each row of $\boldsymbol{X}_{k}(i)$. After spread, each symbol is modulated by the $M \times$ $M$ precoding matrix $\boldsymbol{G}$, which represents an arbitrary linear transform.

In order to avoid interblock interference (IBI) at the receiver, a guard interval of length $L_{g}$ is inserted, $L_{g}$ must be at least the channel order. This operation is represented by a $P \times M$ matrix $\boldsymbol{T}$, where $P=M+L_{g}$.

Finally, each block is serialized and transmitted through antenna $T_{x 1}$ or $T_{x 2}$. The channel impulse response from the $j$ th $(j=1,2)$ transmitter to the $n$-th $(n=1,2, \cdots, N)$ antenna in the receiver, $\boldsymbol{h}_{j n}(i)$, is modelled here as a FIR filter with $L$ taps whose gains are samples of the channel impulse response complex envelope. Assuming that during two symbol periods each multipath channel impulse response remains constant, that is, $\boldsymbol{h}_{j n}(2 i)=\boldsymbol{h}_{j n}(2 i+1)=\left[h_{j n, 0}(2 i) \ldots h_{j n, L-1}(2 i)\right]^{T}$, $(j=1,2, n=1, \cdots, N)$ the transmission through the multipath channel can be represented by a $P \times P$ lower triangular Toeplitz convolution matrix $\boldsymbol{H}_{j n}(2 i)$, whose first column is $\left[h_{j n, 0}(2 i) \ldots h_{j n, L-1}(2 i) \quad 0 \quad \ldots .00\right]^{T}$.

Before detection, at the receiver, the guard interval is processed by the matriz $\boldsymbol{R}$. As we consider a downlink scenario, where the signal for the $K$ users experience the same channel conditions, the received vectors collected on the $n$-th antenna over two consecutive symbol periods are

$$
\begin{aligned}
\boldsymbol{y}_{n}(2 i)= & \boldsymbol{R} \boldsymbol{H}_{1 n}(2 i) \boldsymbol{T} \boldsymbol{G} \sum_{k=1}^{K} \sqrt{\rho_{k}} \boldsymbol{c}_{1 k} s_{k}(2 i) \\
+ & \boldsymbol{R} \boldsymbol{H}_{2 n}(2 i) \boldsymbol{T} \boldsymbol{G} \sum_{k=1}^{K} \sqrt{\rho_{k}} \boldsymbol{c}_{2 k} s_{k}(2 i+1) \\
+ & \boldsymbol{n}_{n}(2 i) \\
\boldsymbol{y}_{n}(2 i+1)= & -\boldsymbol{R} \boldsymbol{H}_{1 n}(2 i) \boldsymbol{T} \boldsymbol{G} \sum_{k=1}^{K} \sqrt{\rho_{k}} \boldsymbol{c}_{1 k} s_{k}^{*}(2 i+1) \\
& +\boldsymbol{R H}_{2 n}(2 i) \boldsymbol{T} \boldsymbol{G} \sum_{k=1}^{K} \sqrt{\rho_{k}} \boldsymbol{c}_{2 k} s_{k}^{*}(2 i) \\
& +\boldsymbol{n}_{n}(2 i+1)
\end{aligned}
$$

where $\boldsymbol{n}_{n}(i)$ is a complex white Gaussian noise vector whose covariance matrix E $\left[\boldsymbol{n}_{n}(i) \boldsymbol{n}_{n}^{H}(i)\right]=\sigma^{2} \boldsymbol{I}_{P}$. The operators $(\cdot)^{H}$ and $\mathrm{E}[\cdot]$ stands for Hermitian transpose and ensemble average, respectively.

Finally, the space-time decoding is performed first by stacking all the column vectors $\boldsymbol{y}_{n}(2 i)$ and $\boldsymbol{y}_{n}^{*}(2 i+1), n=$ $1, \cdots, N$.

\section{A. Particular Cases and Model Simplification}

There are usually four choices for the matrices $G, T$ and $\boldsymbol{R}$, leading to four systems whose parameters are summarized in Table 1, where $\boldsymbol{F}_{M}$ is a $M \times M$ matrix that implements a normalized $M$-point discrete Fourier transform, such that, $\boldsymbol{F}_{M}^{H} \boldsymbol{F}_{M}=\boldsymbol{F}_{M} \boldsymbol{F}_{M}^{H}=\boldsymbol{I}_{M}$,

$$
\boldsymbol{T}_{z p}=\left[\begin{array}{c}
\boldsymbol{I}_{M} \\
\mathbf{0}_{L_{g} \times M}
\end{array}\right] \quad \boldsymbol{T}_{c p}=\left[\begin{array}{c}
\mathbf{0}_{L_{g} \times M-L_{g}} \mid \boldsymbol{I}_{L_{g}} \\
\boldsymbol{I}_{M}
\end{array}\right]
$$

where $\mathbf{0}_{m \times n}$ represents an $m \times n$ null matrix, $\boldsymbol{R}_{z p}=\boldsymbol{I}_{P}$ and $\boldsymbol{R}_{c p}=\left[\mathbf{0}_{M \times L_{g}} \mid \boldsymbol{I}_{M}\right]$.

The use of cyclic prefix or zero padding as guard intervals is interesting because they allow to simplify the model by using the following properties: 
TABLE I

BLOCK TRANSMISSION SYSTEM CONSIDERED

\begin{tabular}{l|c|c|c}
\hline Transmission system & $\boldsymbol{G}$ & $\boldsymbol{T}$ & $\boldsymbol{R}$ \\
\hline SC CDMA CP & $\boldsymbol{I}_{M}$ & $\boldsymbol{T}_{c p}$ & $\boldsymbol{R}_{c p}$ \\
SC CDMA ZP & $\boldsymbol{I}_{M}$ & $\boldsymbol{T}_{z p}$ & $\boldsymbol{R}_{z p}$ \\
MC CDMA CP & $\boldsymbol{F}_{M}^{H}$ & $\boldsymbol{T}_{c p}$ & $\boldsymbol{R}_{c p}$ \\
MC CDMA ZP & $\boldsymbol{F}_{M}^{H}$ & $\boldsymbol{T}_{z p}$ & $\boldsymbol{R}_{z p}$ \\
\hline
\end{tabular}

p1) CP case: $\boldsymbol{R}_{c p} \boldsymbol{H}_{j n}(2 i) \boldsymbol{T}_{c p}$ reduces to a circulant matrix $\mathbb{H}_{j n}(2 i)$ of dimension $M \times M$.

p2) ZP case: $\boldsymbol{R}_{z p} \boldsymbol{H}_{j n}(2 i) \boldsymbol{T}_{z p}$ is equivalent to $\mathbb{H}_{j n}(2 i) \boldsymbol{T}_{z p}$ where $\mathrm{H}_{j n}(2 i)$ is a circulant matrix of dimension $P \times P$. The equivalence is due to $\boldsymbol{R}_{z p}=\boldsymbol{I}_{P}$ and the structure of $T_{z p}$.

p3) A circulant matrix $\mathbb{H}_{j n}(2 i)$ of dimension $Q \times Q$, as in p1) and p2), can be decomposed as $\mathbb{H}_{j n}(2 i)=$ $\boldsymbol{F}_{Q}^{H} \boldsymbol{\Lambda}_{j n}(2 i) \boldsymbol{F}_{Q}$ and $\mathbb{H}_{j n}^{H}(2 i)=\boldsymbol{F}_{Q}^{H} \boldsymbol{\Lambda}_{j n}^{*}(2 i) \boldsymbol{F}_{Q}$, where $\boldsymbol{\Lambda}_{j n}(2 i)$ is a diagonal matrix whose entries are the frequency response of the transmission channel $\boldsymbol{h}_{j n}(2 i)$, i.e., $\boldsymbol{\Lambda}_{j n}(2 i)=\operatorname{diag}\left(\tilde{\boldsymbol{F}}_{Q \times L} \boldsymbol{h}_{j n}(2 i)\right)$, where $\tilde{\boldsymbol{F}}_{Q \times L}$ is a $Q \times L$ matrix formed with the first $L$ columns of the matrix that implements the (non-normalized) $Q$-point FFT.

Then, for ZP case

$$
\begin{aligned}
\boldsymbol{R H}_{j n}(2 i) \boldsymbol{T} \boldsymbol{G} \boldsymbol{c}_{j k} & =\mathbb{H}_{j n}(2 i) \boldsymbol{T}_{Z P} \boldsymbol{G} \boldsymbol{c}_{j k} \\
& =\boldsymbol{F}_{P}^{H} \boldsymbol{\Lambda}_{j n}(2 i) \boldsymbol{F}_{P} \boldsymbol{T}_{Z P} \boldsymbol{G} \boldsymbol{c}_{j k} \\
& =\boldsymbol{F}_{P}^{H} \operatorname{diag}\left(\boldsymbol{F}_{P} \boldsymbol{T}_{Z P} \boldsymbol{G} \boldsymbol{c}_{j k}\right) \tilde{\boldsymbol{F}}_{P \times L} \boldsymbol{h}_{j n}(2 i) \\
& =\boldsymbol{V}_{j k}^{Z P} \boldsymbol{h}_{j n}(2 i)
\end{aligned}
$$

and for $\mathrm{CP}$ case:

$$
\begin{aligned}
\boldsymbol{R H}_{j n}(2 i) \boldsymbol{T} \boldsymbol{G} \boldsymbol{c}_{j k} & =\mathbb{H}_{j n}(2 i) \boldsymbol{G} \boldsymbol{c}_{j k} \\
& =\boldsymbol{F}_{M}^{H} \boldsymbol{\Lambda}_{j n}(2 i) \boldsymbol{F}_{M} \boldsymbol{G} \boldsymbol{c}_{j k} \\
& =\boldsymbol{F}_{M}^{H} \operatorname{diag}\left(\boldsymbol{F}_{M} \boldsymbol{G} \boldsymbol{c}_{j k}\right) \tilde{\boldsymbol{F}}_{M \times L} \boldsymbol{h}_{j n}(2 i) \\
& =\boldsymbol{V}_{j k}^{C P} \boldsymbol{h}_{j n}(2 i)
\end{aligned}
$$

From (2) and (3) it follows that,

$$
\begin{aligned}
\boldsymbol{y}_{n}(2 i)= & \sum_{k=1}^{K} \sqrt{\rho_{k}}\left[\boldsymbol{V}_{1 k} \boldsymbol{h}_{1 n}(2 i) s_{k}(2 i)\right. \\
& \left.+\boldsymbol{V}_{2 k} \boldsymbol{h}_{2 n}(2 i) s_{k}(2 i+1)\right]+\boldsymbol{n}_{n}(2 i) \\
\boldsymbol{y}_{n}(2 i+1)= & \sum_{k=1}^{K} \sqrt{\rho_{k}}\left[-\boldsymbol{V}_{1 k} \boldsymbol{h}_{1 n}(2 i) s_{k}^{*}(2 i+1)\right. \\
& \left.+\boldsymbol{V}_{2 k} \boldsymbol{h}_{2 n}(2 i) s_{k}^{*}(2 i)\right]+\boldsymbol{n}_{n}(2 i+1)
\end{aligned}
$$

where $\boldsymbol{V}_{j k}$ is a code related matrix of dimension $Q \times L, \boldsymbol{V}_{j k}=$ $\boldsymbol{V}_{j k}^{C P}, Q=M$ for $\mathrm{CP}$ case and $\boldsymbol{V}_{j k}=\boldsymbol{V}_{j k}^{Z P}, Q=P$ for $\mathrm{ZP}$ case.

Stacking all the column vectors $\boldsymbol{y}_{n}(2 i)$ and $\boldsymbol{y}_{n}^{*}(2 i+1)$, $n=1, \cdots, N$, we get the observation vector

$$
\begin{aligned}
\boldsymbol{r}(i)= & {\left[\begin{array}{c}
\boldsymbol{y}_{1}(2 i) \\
\boldsymbol{y}_{1}^{*}(2 i+1) \\
\vdots \\
\boldsymbol{y}_{N}(2 i) \\
\boldsymbol{y}_{N}^{*}(2 i+1)
\end{array}\right] } \\
= & \sum_{k=1}^{K} \sqrt{\rho_{k}}\left\{\overline{\boldsymbol{\Psi}}_{k} \boldsymbol{h}(i) s_{k}(2 i)+\widetilde{\boldsymbol{\Psi}}_{k} \boldsymbol{h}^{*}(i) s_{k}(2 i+1)\right\} \\
& +\overline{\boldsymbol{n}}(i)
\end{aligned}
$$

where

$$
\boldsymbol{h}(i)=\left[\begin{array}{c}
\boldsymbol{h}_{11}(2 i) \\
\boldsymbol{h}_{21}^{*}(2 i) \\
\vdots \\
\boldsymbol{h}_{1 N}(2 i) \\
\boldsymbol{h}_{2 N}^{*}(2 i)
\end{array}\right]
$$

is a $2 L N$ dimensional vector which we call the composed channel and

$$
\begin{aligned}
& \overline{\boldsymbol{\Psi}}_{k}=\boldsymbol{I}_{N} \otimes\left[\begin{array}{cc}
\boldsymbol{V}_{1 k} & \mathbf{0}_{Q \times L} \\
\mathbf{0}_{Q \times L} & \boldsymbol{V}_{2 k}^{*}
\end{array}\right] \\
& \widetilde{\boldsymbol{\Psi}}_{k}=\boldsymbol{I}_{N} \otimes\left[\begin{array}{cc}
\mathbf{0}_{Q \times L} & \boldsymbol{V}_{2 k} \\
-\boldsymbol{V}_{1 k}^{*} & \mathbf{0}_{Q \times L}
\end{array}\right]
\end{aligned}
$$

where $\otimes$ is defined as the Kronecker product.

\section{Linearly CONSTRAined Minimum VARIANCE RECEIVERS}

In the following we assume without lost of generality that user one is desired and drop the user index, $k$. The design of the receiver filters $\boldsymbol{W}=\left[\begin{array}{ll}\overline{\boldsymbol{w}} & \widetilde{\boldsymbol{w}}\end{array}\right] \in \mathbb{C}^{2 Q N \times 2}$, based on the minimum variance (MV) criterion uses the output energy as a cost function to be minimized:

$$
\begin{aligned}
\boldsymbol{J}_{M V}(\boldsymbol{W}) & =\mathrm{E}\left[\left\|\boldsymbol{W}^{H} \boldsymbol{r}(i)\right\|^{2}\right] \\
& =\operatorname{tr}\left[\boldsymbol{W}^{H} \boldsymbol{R}_{\boldsymbol{r} \boldsymbol{r}} \boldsymbol{W}\right] \\
& =\overline{\boldsymbol{w}}^{H} \boldsymbol{R}_{\boldsymbol{r} \boldsymbol{r}} \overline{\boldsymbol{w}}+\widetilde{\boldsymbol{w}}^{H} \boldsymbol{R}_{\boldsymbol{r} \boldsymbol{r}} \widetilde{\boldsymbol{w}}
\end{aligned}
$$

where $\boldsymbol{R}_{\boldsymbol{r} \boldsymbol{r}}=\mathrm{E}\left[\boldsymbol{r}(i) \boldsymbol{r}^{H}(i)\right]$ and $\operatorname{tr}[\cdot]$ stands for trace.

In order to avoid the trivial solution, $\boldsymbol{W}=\mathbf{0}$ and anchor the desired user signal, $\boldsymbol{W}$ is subject to a set of constraints

$$
\begin{array}{r}
\overline{\boldsymbol{\Psi}}^{H} \overline{\boldsymbol{w}}=\hat{\boldsymbol{h}} \\
\widetilde{\boldsymbol{\Psi}}^{H} \widetilde{\boldsymbol{w}}=\hat{\boldsymbol{h}}^{*}
\end{array}
$$

where $\hat{\boldsymbol{h}}$ is an estimate of the composed channel (7).

Using the method of Lagrange multipliers, the optimum receiver vector is calculated as

$$
\begin{gathered}
\overline{\boldsymbol{w}}_{o p t}=\boldsymbol{R}_{\boldsymbol{r} \boldsymbol{r}}^{-1} \overline{\boldsymbol{\Psi}}\left(\overline{\boldsymbol{\Psi}}^{H} \boldsymbol{R}_{\boldsymbol{r} \boldsymbol{r}}^{-1} \overline{\boldsymbol{\Psi}}\right)^{-1} \hat{\boldsymbol{h}} \\
\widetilde{\boldsymbol{w}}_{o p t}=\boldsymbol{R}_{\boldsymbol{r} \boldsymbol{r}}^{-1} \widetilde{\boldsymbol{\Psi}}\left(\widetilde{\boldsymbol{\Psi}}^{H} \boldsymbol{R}_{\boldsymbol{r} \boldsymbol{r}}^{-1} \widetilde{\boldsymbol{\Psi}}\right)^{-1} \hat{\boldsymbol{h}}^{*}
\end{gathered}
$$

and the symbols are estimated as $\hat{s}(2 i)=\operatorname{disc}\left\{\overline{\boldsymbol{w}}_{\text {opt }}^{H} \boldsymbol{r}(i)\right\}$ and $\hat{s}(2 i+1)=\operatorname{disc}\left\{\widetilde{\boldsymbol{w}}_{o p t}^{H} \boldsymbol{r}(i)\right\}$, where the $\operatorname{disc}\{x\}$ is the symbol of the signal constellation closer to $x$.

The channel estimate can be obtained following a max/min approach as in [6], [13], i.e., maximizing the minimum output 
variance after interference reduction. In order to reduce the computational complexity we follow the approach in [9], then the resulting output variance is

$$
\boldsymbol{J}_{M V}\left(\boldsymbol{W}_{\text {opt }}\right)=\hat{\boldsymbol{h}}^{H} \overline{\boldsymbol{Q}}^{H} \boldsymbol{R}_{\boldsymbol{r} \boldsymbol{r}} \overline{\boldsymbol{Q}} \hat{\boldsymbol{h}}+\hat{\boldsymbol{h}}^{T} \widetilde{\boldsymbol{Q}}^{H} \boldsymbol{R}_{\boldsymbol{r} \boldsymbol{r}} \widetilde{\boldsymbol{Q}} \hat{\boldsymbol{h}}^{*},
$$

and

$$
\hat{\boldsymbol{h}}_{\text {opt }}=\arg \max _{\|\hat{\boldsymbol{h}}\|=1} \boldsymbol{J}_{M V}\left(\boldsymbol{W}_{\text {opt }}\right)
$$

where

$$
\begin{aligned}
\overline{\boldsymbol{Q}} & =\boldsymbol{R}_{\boldsymbol{r} \boldsymbol{r}}^{-1} \overline{\mathbf{\Psi}}\left(\overline{\boldsymbol{\Psi}}^{H} \boldsymbol{R}_{\boldsymbol{r} \boldsymbol{r}}^{-1} \overline{\mathbf{\Psi}}\right)^{-1} \\
\widetilde{\boldsymbol{Q}} & =\boldsymbol{R}_{\boldsymbol{r} \boldsymbol{r}}^{-1} \widetilde{\boldsymbol{\Psi}}\left(\widetilde{\boldsymbol{\Psi}}^{H} \boldsymbol{R}_{\boldsymbol{r} \boldsymbol{r}}^{-1} \widetilde{\mathbf{\Psi}}\right)^{-1}
\end{aligned}
$$

Using the fact that $\hat{\boldsymbol{h}}^{T} \widetilde{\boldsymbol{Q}}^{H} \boldsymbol{R}_{\boldsymbol{r} \boldsymbol{r}} \widetilde{\boldsymbol{Q}} \hat{\boldsymbol{h}}^{*}$ is real valued and taking into account the conjugate symmetric properties induced by space-time block codes [6], $\hat{\boldsymbol{h}}$ can be estimated by

$$
\begin{aligned}
\hat{\boldsymbol{h}}_{\text {opt }} & =\arg \max _{\|\hat{\boldsymbol{h}}\|=1} \hat{\boldsymbol{h}}^{H}\left(\overline{\boldsymbol{Q}}^{H} \boldsymbol{R}_{\boldsymbol{r} \boldsymbol{r}} \overline{\boldsymbol{Q}}\right) \hat{\boldsymbol{h}} \\
& =\arg \max _{\|\hat{\boldsymbol{h}}\|=1} \hat{\boldsymbol{h}}^{H} \boldsymbol{R}_{\boldsymbol{r}_{u} \boldsymbol{r}_{u}} \hat{\boldsymbol{h}}
\end{aligned}
$$

where $\boldsymbol{R}_{\boldsymbol{r}_{u} \boldsymbol{r}_{u}}=\mathrm{E}\left[\boldsymbol{r}_{u}(i) \boldsymbol{r}_{u}^{H}(i)\right]$ and $\boldsymbol{r}_{u}(i)=\overline{\boldsymbol{Q}}^{H} \boldsymbol{r}(i)$. The solution of (19) is the eigenvector corresponding to the maximum eigenvalue of $\boldsymbol{R}_{\boldsymbol{r}_{u} \boldsymbol{r}_{u}}$.

\section{A RECURSIVE LEAST SQUARES IMPLEMENTATION}

In order to avoid the high computational cost associated with direct inverse of an estimative of $\boldsymbol{R}_{\boldsymbol{r} r}$, necessary to compute (13)-(14), a recursive least squares solution that uses Kalman RLS recursions can be employed. The update formulae are as follows. First compute

$$
\hat{\boldsymbol{R}}_{\boldsymbol{r} \boldsymbol{r}}^{-1}(i)=\frac{1}{\lambda}\left[\hat{\boldsymbol{R}}_{\boldsymbol{r} \boldsymbol{r}}^{-1}(i-1)-\gamma(i) \boldsymbol{\psi}(i) \boldsymbol{\psi}^{H}(i-1)\right],
$$

where $\boldsymbol{\psi}(i)$ is defined as the Kalman gain vector, $\boldsymbol{\psi}(i)=$ $\hat{\boldsymbol{R}}_{\boldsymbol{r} \boldsymbol{r}}^{-1}(i-1) \boldsymbol{r}(i), \gamma(i)=\left[\frac{\lambda}{1-\lambda}+\boldsymbol{r}^{H}(i) \hat{\boldsymbol{R}}_{\boldsymbol{r} \boldsymbol{r}}^{-1}(i-1) \boldsymbol{r}(i)\right]^{-1}$ and $0<\lambda<1$ is the forgetting factor.

Then, post-multiplying (20) by $\overline{\boldsymbol{\Psi}}, \overline{\boldsymbol{\Gamma}}(i)=\boldsymbol{R}_{\boldsymbol{r} \boldsymbol{r}}^{-1}(i) \overline{\boldsymbol{\Psi}}$ can be updated as [14]:

$$
\overline{\boldsymbol{\Gamma}}(i)=\frac{1}{\lambda}\left[\overline{\boldsymbol{\Gamma}}(i-1)-\gamma(i) \boldsymbol{\Psi}(i) \boldsymbol{r}^{H}(i) \overline{\boldsymbol{\Gamma}}(i-1)\right]
$$

The value for the input signal autocorrelation matrix at time zero is [14]

$$
\hat{\boldsymbol{R}}_{\boldsymbol{r} \boldsymbol{r}}(0)=E_{0} \operatorname{diag}\left(1, \lambda^{-1}, \lambda^{-2}, \cdots, \lambda^{-(M-1)}\right)
$$

where $E_{0}$ is the forward prediction error energy and must be a positive value. At instant zero, $\overline{\boldsymbol{\Gamma}}(0)=\boldsymbol{R}_{\boldsymbol{r} \boldsymbol{r}}^{-1}(0) \overline{\boldsymbol{\Psi}}$.

Now, using (21), applying the matrix inversion lemma to $\overline{\boldsymbol{\Psi}}^{H} \overline{\boldsymbol{\Gamma}}(i)$ and pre-multiplying the result by $\overline{\boldsymbol{\Gamma}}(i)$ we get that $\hat{\overline{\boldsymbol{Q}}}(i)=\overline{\boldsymbol{\Gamma}}(i)\left(\overline{\boldsymbol{\Psi}}^{H} \overline{\boldsymbol{\Gamma}}(i)\right)^{-1}$ can be estimated recursively as [14]:

$$
\begin{aligned}
\overline{\boldsymbol{u}}(i)= & \overline{\boldsymbol{\Psi}}^{H} \boldsymbol{\psi}(i) \\
\overline{\boldsymbol{v}}^{H}(i)= & \boldsymbol{r}^{H}(i) \hat{\overline{\boldsymbol{Q}}}(i-1) \\
\hat{\overline{\boldsymbol{Q}}}(i)= & {\left[\hat{\overline{\boldsymbol{Q}}}(i-1)-\gamma(i) \boldsymbol{\psi}(i) \overline{\boldsymbol{v}}^{H}(i)\right] . } \\
& \cdot\left[\boldsymbol{I}_{2 N L}+\frac{\overline{\boldsymbol{u}}(i) \overline{\boldsymbol{v}}^{H}(i)}{\frac{1}{\gamma(i)}-\overline{\boldsymbol{v}}^{H}(i) \overline{\boldsymbol{u}}(i)}\right],
\end{aligned}
$$

and the initialization is performed as $\hat{\bar{Q}}(0)=$ $\overline{\boldsymbol{\Gamma}}(0)\left(\overline{\boldsymbol{\Psi}}^{H} \overline{\boldsymbol{\Gamma}}(0)\right)^{-1}$. Similar formulae can be follow in order to estimate $\widetilde{\boldsymbol{Q}}(i)$.

To estimate the channel vector using (19) without incurring into the high computational cost of the singular value decomposition (SVD), a subspace tracking algorithm can be used. As proposed in [9], we use the PASTd algorithm [15], which tracks the principal component sequentially and is ideally suited when only the largest eigenvalue and the corresponding eigenvector of a rank-one update matrix $\left(\boldsymbol{R}_{\boldsymbol{r}_{u} \boldsymbol{r}_{u}}\right)$ are needed, as in this case. The overall algorithm for channel and receiver estimator is summarized in Tab. II.

TABLE II

CHANNEL AND RECEIVER ESTIMATION.

$$
\begin{aligned}
& \text { a) Update } \hat{\overline{\boldsymbol{Q}}}(i) \text { and } \hat{\widetilde{\boldsymbol{Q}}}(i) \text { as in (23)-(25) } \\
& \text { b) Compute } \overline{\boldsymbol{r}}_{u}(i)=\hat{\overline{\boldsymbol{Q}}}^{H}(i) \boldsymbol{r}(i) \\
& \text { c) Update the channel estimate using the PASTd algorithm: } \\
& \qquad \begin{array}{l}
\quad \beta(i)=\hat{\boldsymbol{h}}^{H}(i-1) \overline{\boldsymbol{r}}_{u}(i) \\
\quad \alpha(i)=\lambda \alpha(i-1)+|\boldsymbol{\beta}(i)|^{2} \\
\quad \hat{\boldsymbol{h}}(i)=\hat{\boldsymbol{h}}(i-1)+\left(\overline{\boldsymbol{r}}_{u}(i)-\hat{\boldsymbol{h}}(i-1) \beta(i)\right) \beta^{*}(i) / \alpha(i) \\
\text { d) Compute the filters } \overline{\boldsymbol{w}}(i)=\hat{\overline{\boldsymbol{Q}}}(i) \hat{\boldsymbol{h}}(i) \text { and } \widetilde{\boldsymbol{w}}(i)=\hat{\widetilde{Q}}(i) \hat{\boldsymbol{h}}^{*}(i)
\end{array}
\end{aligned}
$$

\section{Simulation Results}

The simulation results presented are for a BPSK synchronous single carrier and multicarrier CDMA systems that employ cyclic prefix and zero padding as guard intervals. All systems assume a guard interval length of $L_{g}=3$. Because we focus on a downlink scenario, users experience the same channel conditions. For fading channels, the sequence of channel coefficients, $h_{j n, l}(i)=p_{j n, l} \alpha_{j n, l}(i)(l=0,1,2, \ldots, L-1$, $j=1,2, n=1, \cdots N)$ is obtained with Clarke's model [16], where it is assumed that $L=4$. The channel is time-varying with $f_{d} T=0.0001$ in all cases. We use $N=1$ antenna at the receiver. Results are measured after convergence of the filters and are the average of 500 ensembles.

In Fig. 2 - Fig. 4 we assess the desired user BER performance of the analyzed receivers versus $E_{b} / N_{0}$ for different spreading codes. The first one employs pseudo-noise (PN) sequences of length $M=15$ as spreading codes, the second employs Zadoff-Chu [17] [18] codes of length $M=16$ and finally the third employs Hadamard sequences of length $M=16$. The system has $K=4$ users. Regarding power distribution, we simulate a severe near-far scenario where each interferer has a power level $10 \mathrm{~dB}$ above the desired user, that is, near-far ratio $(N F R)$ is equal to $10 \mathrm{~dB}$.

In the next experiment we assess the desired user BER performance of the analyzed receivers versus the number of users, $K$ for a fixed signal-to-noise ratio. The desired user has $E_{b} / N_{0}=15 \mathrm{~dB}$ and its $N F R=10 \mathrm{~dB}$. We employ two user codes: pseudo-noise $(M=15))$ and Zadoff-Chu $(M=16)$, results are shown in Fig. 5 and Fig. 6.

Finally, we plot the desired user BER performance versus the near-far ratio for a fixed number of users, $K=4$, and for a fixed signal-to-noise ratio. The desired user has $E_{b} / N_{0}=$ $15 \mathrm{~dB}$. As before, we use two user codes: pseudo-noise $(M=$ 
15)) and Zadoff-Chu $(M=16)$, results are shown in Fig. 7 and Fig. 8 .

As can be seen, the performance in terms of bit error rate for each system varies according to the type of the user code used. In the case of using Hadamard codes, note that SC CDMA CP and SC CDMA ZP systems degrade due to the ill-conditioned matrices used for channel estimation, formed with several choices of Hadamard user codes. MC CDMA $\mathrm{CP}$ suffer a strong degradation if Zadoff-Chu are used, but it presents the best performance with Hadamard or PN codes. Note the floor on the bit error rate for $E_{b} / N_{0} \geq 18 \mathrm{~dB}$. This is due mainly to channel fading and errors in the channel estimate.

Regarding the robustness to system load and near-far effect, we also note the degradation of MC CDMA CP resulting when Zadoff-Chu codes are used. Results for the cases where the guard interval is greater than the channel order and scenarios where $N=2$ antennas are used at the receiver can be found in [19].

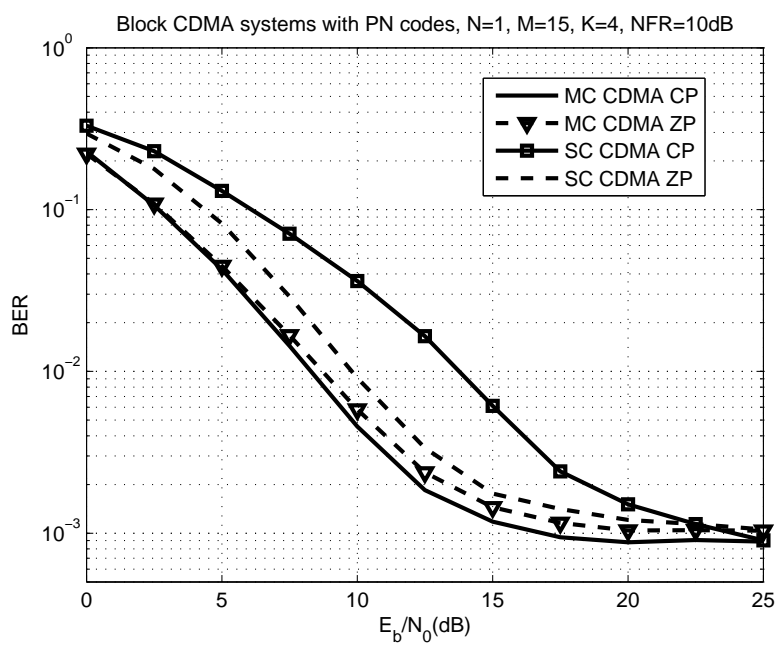

Fig. 2. BER versus $E_{b} / N_{0}(\mathrm{~dB}), \mathrm{PN}$ codes.

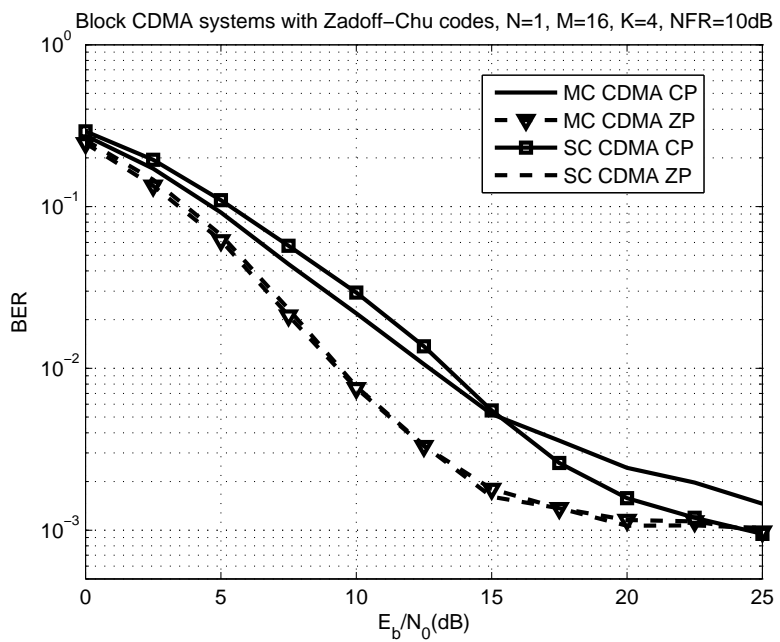

Fig. 3. BER versus $E_{b} / N_{0}(\mathrm{~dB})$, Zadoff-Chu codes.

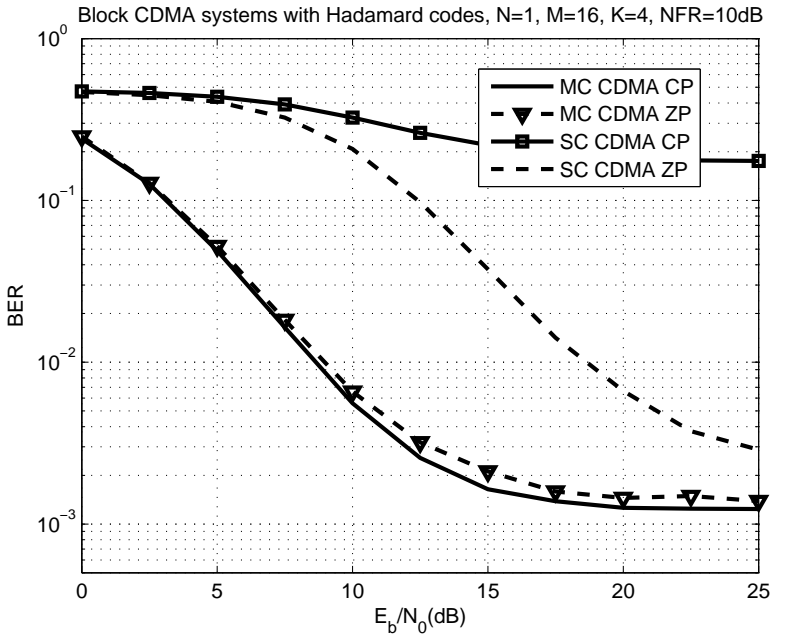

Fig. 4. BER versus $E_{b} / N_{0}(\mathrm{~dB})$, Hadamard codes.

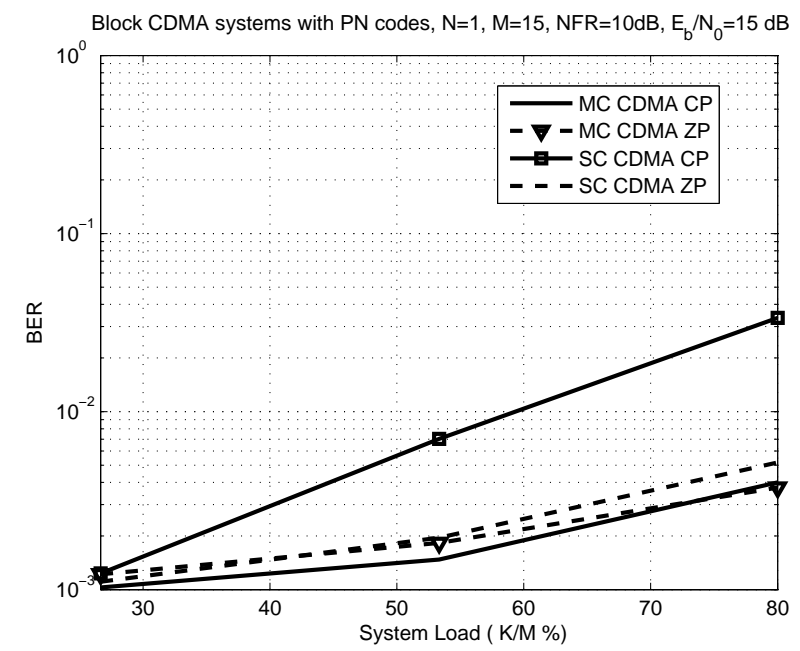

Fig. 5. BER versus System $\operatorname{Load}(K / M \%)$, PN codes.

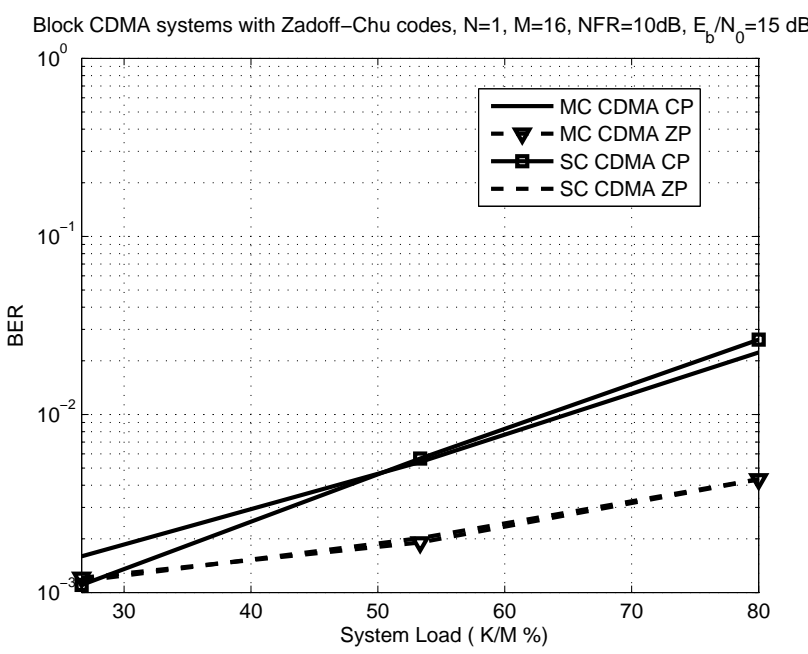

Fig. 6. BER versus System $\operatorname{Load}(K / M \%)$, Zadoff-Chu codes.

\section{CONCLUSIONS}

In this work we propose a space-time block CDMA transmission systems in single or multiple carrier modulation. We 


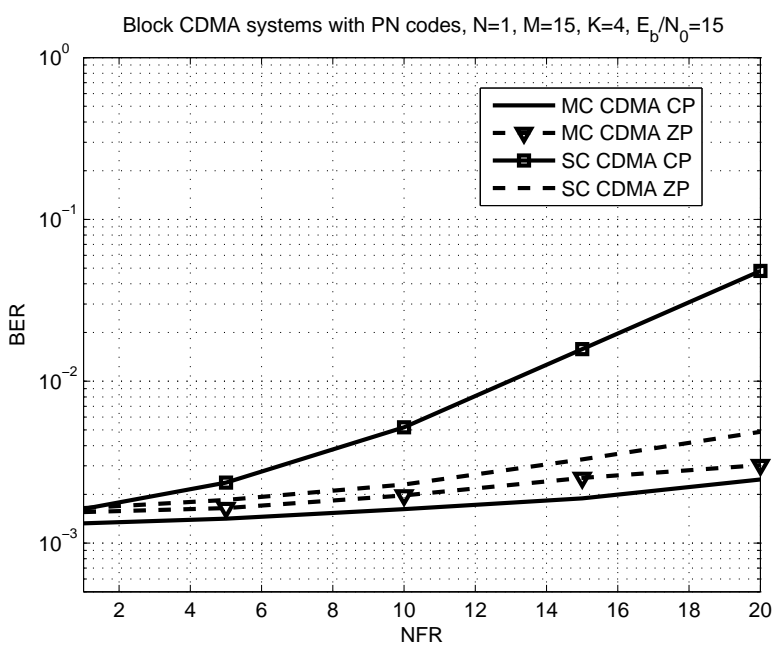

Fig. 7. BER versus $N F R(\mathrm{~dB})$, PN codes.

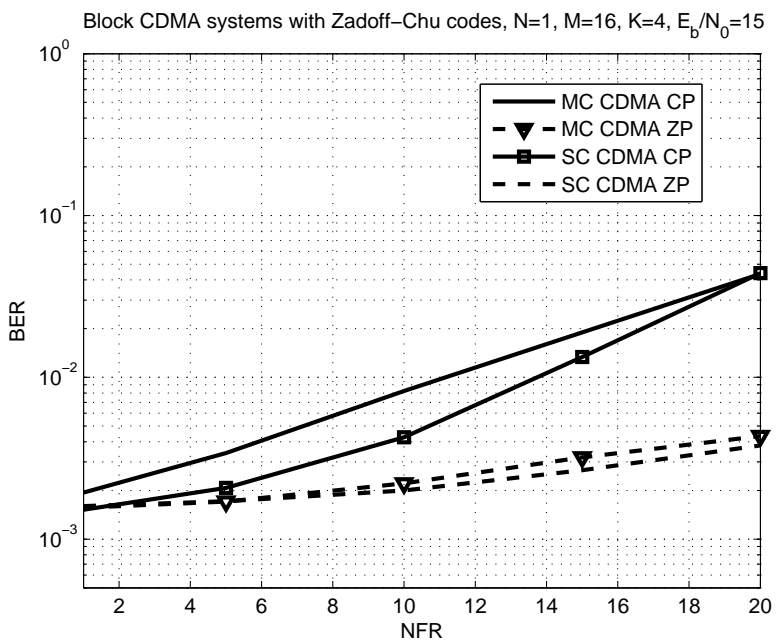

Fig. 8. BER versus NFR (dB), Zadoff-Chu codes.

also provide an unified framework for analise and comparison between these systems and a blind receiver based on the minimum variance criterion was implemented. It was shown through computer simulations that under different choices of spreading codes, system performance varies in terms of BER, robustness to system load and near-far effect.

\section{REFERENCES}

[1] A. Greenspan, M. Klerer, J. Tomcik, R. Canchi, and J. Wilson, "IEEE 802.20: Mobile broadband wireless access for the twenty-first century," IEEE Commun. Mag., vol. 46, no. 7, pp. 56-63, july 2008.

[2] A. Chouly, A. Brajal, and S. Jourdan, "Orthogonal multicarrier techniques applied to direct sequence spread spectrum CDMA systems," Proc. GLOBECOM, pp. 1723-1728, november 1993.

[3] V. Dasilva and E. S. Sousa, "Performance of orthogonal CDMA codes for quasi-synchronous communication systems," Proc. ICUPC, pp. 995999, october 1993.

[4] K. L. Baum, T. A. Thomas, F. W. Vook, and V. Nangia, "Cyclic-prefix CDMA: an improved transmission method for broadband DS-CDMA cellular systems," Proc. WCNC, vol. 1, pp. 183-188, march 2002.

[5] A. S. Madhukumar, F. Chin, Y-C. Liang, and K. Yang, "Single-carrier cyclic prefix-assisted CDMA system with frequency domain equalization for high data rate transmission," EURASIP J. Wirel. Commun. Netw., vol. 2004, no. 1, pp. 149-160, 2004.
[6] H. Li, X. Lu, and G. B. Giannakis, "Capon multiuser receiver for CDMA systems with space-time coding," IEEE Trans. Signal Processing, vol. 50, no. 5, pp. 1193-1204, may 2002.

[7] R. C. de Lamare and R. Sampaio-Neto, "Blind adaptive space-time block-coded receivers for DS-CDMA systems in multipath channels based on the constant modulus criterion," Proc. VI International Telecommunications Symposium, pp. 449-454, september 2006.

[8] C. A. Medina, T. T. V. Vinhoza, and R. Sampaio-Neto, "Performance comparison of space-time block coding for single and multicarrier CDMA systems," XXV Simpósio Brasileiro de Telecomunicações (SBrT 2007), Recife, Brazil, september 2007.

[9] C. A. Medina, T. T. V. Vinhoza, and R. Sampaio-Neto, "A blind channel estimation algorithm for space-time coded MC-CDMA receivers," Accepted in IEEE International Conference on Communications (ICC2009), Dresden, German, june 2009.

[10] M. Steer, "Beyond 3G," IEEE Microwave, vol. 8, no. 1, pp. 76-82, february 2007.

[11] S. Barbarossa, Multiantenna Wireless Communication Systems, Artech House, 2005.

[12] S. Alamouti, "A simple transmit diversity technique for wireless communications," IEEE J. Select. Areas Commun., vol. 16, no. 8, october 1998.

[13] Z. Xu and M. K. Tsatsanis, "Blind adaptive algorithms for minimum variance CDMA receivers," IEEE Trans. Commun., vol. 49, no. 1, pp. 180-194, january 2001.

[14] L. S. Resende, J. M. Romano, and M. Bellanger, "A fast least-squares algorithm for linearly constrained adaptive filtering," IEEE Trans. Signal Processing, vol. 44, no. 5, pp. 1168-1174, may 1996.

[15] X. Wang and H. V. Poor, Wireless Communication Systems Advanced Techniques for Signal Reception, Prentice-Hall, 2003.

[16] T. S. Rappaport, Wireless Communications: Principles and Practice, Prentice Hall PTR, 1996.

[17] R. L. Frank and S. A. Zadoff, "Phase shift pulse codes with good periodic correlation properties," IEEE Trans. Inform. Theory, vol. IT-8, pp. 381-382, october 1962.

[18] D. Chu, "Polyphase codes with good periodic correlation properties," IEEE Trans. Inform. Theory, vol. 18, no. 4, pp. 531-532, july 1972.

[19] C. A. Medina, Transmissão CDMA por Blocos em Canais SISO e MISO, Ph.D. thesis, Centro de Estudos em Telecomunica cões (CETUC), PUCRio, march 2009. 